\title{
The multi-polar planetary nebula NGC 5189
}

\author{
Laurence Sabin, Roberto Vázquez, Jose A. Lopéz and \\ Maria-Teresa García-Diaz
}

\author{
Instituto de Astronomía, Universidad Nacional Autónoma de México, \\ Apdo. Postal 877, 22800 Ensenada, B.C, Mexico \\ emails: lsabin, vazquez, jal, tere@astrosen.unam.mx
}

\begin{abstract}
NGC 5189 is a particularly interesting planetary nebula (PN) displaying multiple bipolar structures. This type of morphology is generally attributed to multiple mass loss events believed to originate from a precessing central source. In order to better understand this, once dubbed chaotic PN, we have investigated optical imaging combined with low- and high- resolution spectroscopic data to dissect its components. The imaging reveals three and possibly four well defined bipolar lobes showing a misalignment with respect to the central torus, although sharing the same geometric center. The high nitrogen levels detected in the elongated filaments/condensations surrounding the nebula and at the torus location highlight the presence of low ionization structures as well as the possible occurrence of shocks in the areas perturbed by large dynamical motions. Finally the kinematical study indicates moderate expansion velocities $\left(35 \mathrm{~km} . \mathrm{s}^{-1}\right.$ in the western lobe, $33 \mathrm{~km} . \mathrm{s}^{-1}$ in the central region and $44 \mathrm{~km} . \mathrm{s}^{-1}$ in the eastern lobe). We also notice the asymmetry of the [NII] distribution and the velocities inside NGC 5189 between its North-West and South-East components.
\end{abstract}

Keywords. planetary nebulae: individual (NGC 5189)

\section{Introduction and Observations}

NGC 5189 belongs to the group of planetary nebulae (PNe) with multiple bipolar structures whose morphologies have been proposed by Soker and Livio $(1989,1994)$ to be the result of close binary systems combined with a precessing systemic axis. Very few morphological studies have been realised about NGC 5189 e.g. Phillips and Reay (1983) and Reay et al. (1984). The new set of imaging and spectroscopic data that we secured reveals now additional details on the morphological structure of NGC 5189.

Optical images of NGC 5189 were obtained at Las Campanas Observatory (LCO, Chile) on the $1.0 \mathrm{~m}$ Swope telescope and we present in Fig. 1 the $\mathrm{H} \alpha$ and $\mathrm{H} \alpha /[\mathrm{OIII}]$ ones. They indicate the presence of a toroidal structure, mainly composed of knots, with a major axis of about 78 arcsec. Figure 1 also shows the three obvious outflows seen in NGC 5189 and numbered 1, 2 and 3, oriented at the respective position angles (PA) of $90^{\circ}, 37^{\circ}$ and $141^{\circ}$ and the respective pair of lobes A1-A2, B1-B2 and $\mathrm{C} 1-\mathrm{C} 2$. The existence of a fourth bipolar system (numbered 4 on the figure) at PA $10^{\circ}$ is less clear as only the polar caps of the possible ejections are seen (D1 and D2). All four bipolar structures are emerging from the same geometric center related to the central star.

Longslit intermediate dispersion spectra were obtained with the modular spectrometer on the $2.5 \mathrm{~m} \mathrm{du}$ Pont telescope at LCO. Two exposures were realised at the same position angle $\mathrm{PA}=329^{\circ}$ but 36.6 arcsec apart. Along each slit we followed the evolution of the $\mathrm{H} \alpha /[\mathrm{NII}]$ and $\mathrm{H} \alpha /[\mathrm{SII}]$ ratios. The first ratio, $\mathrm{H} \alpha /[\mathrm{NII}]$, behaves in roughly the same way in both slits with a high nitrogen level in the central toroidal structure $\mathrm{H} \alpha /[\mathrm{NII}]<1$ and becoming $>1$ outside this area. We also notice a [NII] increase in the elongated filaments/condensations parallel to the torus that seem to be related to the southern outflow C1 (Fig.1). No opposite counterpart of such structure is detected in the outflow C2. High 

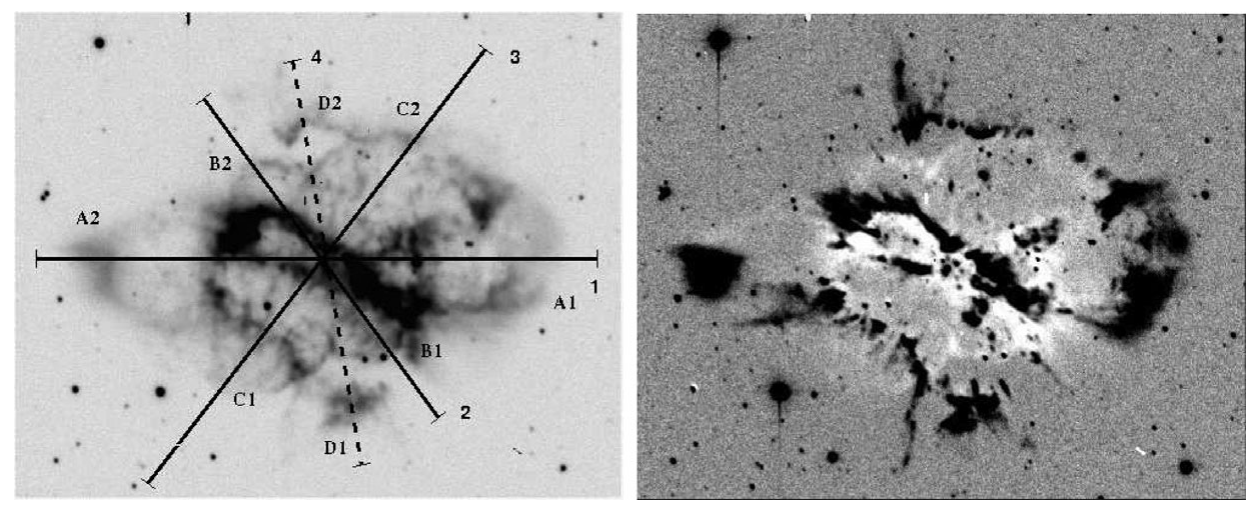

Figure 1. Left: outflows location in the PN NGC 5189. The dash lines indicates a possibly fourth ejection axis. Right: $\mathrm{H} \alpha /[\mathrm{OIII}]$ image ratio highlighting the high excitation zones. Black regions refer to high ratios and white regions refer to low ratios. North is up and East is left in a field of view of $\sim 3.0^{\prime} \times 3.7^{\prime}$.

resolution spectra have been obtained with the Manchester Echelle Spectrometer (MES) on the $3.9 \mathrm{~m}$ Anglo-Australian telescope (AAT). We identify a systemic velocity $\mathrm{V}_{\text {hel }}$ of $9.2 \mathrm{~km} . \mathrm{s}^{-1}$ and the following maximum expansion velocities (cf. Fig. 1, right): $35 \mathrm{~km} \mathrm{~s} \mathrm{~s}^{-1}$ in the western lobe, $33 \mathrm{~km} \mathrm{~s}^{-1}$ in the central region and $44 \mathrm{~km} \mathrm{~s}^{-1}$ in the eastern lobe. Those velocities do not show extreme values as would suggest the complex morphology but they are still higher than the average PN expansion $\left(\sim 25 \mathrm{~km} \mathrm{~s}^{-1}\right)$.

\section{Conclusion}

The high excitation PN NGC 5189 displays three and possibly four sets of bipolar lobes. These lobes seem surrounded by an extended filamentary and knotty envelope. Except for the opposite lobes along the axis numbered 3 in Fig. 1, all the bipolar systems show a misalignment with the torus while sharing the same geometric center. This suggests a precessing/wobbling pattern. The spectroscopic analysis indicates zones of [NII] enrichment mainly in the elongated filaments/condensations that could be related to the local shock environment or LIS. Finally the kinematical study indicates moderate expansion velocities. The data obtained alleviate the previous chaotic view of NGC 5189 by analysing its different components. Full details of this work will soon be published in a forthcoming paper.

\section{Acknowledgements}

This work is supported by PAPIIT-UNAM grant IN109509 (Mexico).

\section{References}

Phillips, J. P. \& Reay, N. K. 1983, A\& A, 117, 33

Reay, N. K., Atherton, P. D., \& Taylor, K. 1984, MNRAS, 206, 71

Soker, N. \& Livio, M. 1994, ApJ, 421, 219

Soker, N. \& Livio, M. 1989, ApJ, 339, 268 